$$
\text { "tmcs-kantor" — 2012/2/27 — 20:51 — page } 77 \text { — \#1 }
$$

\title{
On an international training of mathematically talented students Assets of the 20 years of the "Nagy Károly Mathematical Student-meetings"
}

\author{
SÁNDOR KÁNTOR and TÜNDE KÁNTOR
}

Abstract. The focus of this paper is to present the gems of the "Nagy Károly Mathematical Student-meetings" in Rév-Komárom (Slovakia) from 1991 to 2010. During these 20 years there was done a lot of work to train mathematically talented students with Hungarian mother tongue and to develop their mathematical thinking, and to teach them problem solving and heuristic strategies for successful acting on the competitions. We collected the most interesting problems and methods presented by the trainer teachers.

Key words and phrases: mathematical thinking and knowledge, problem solving, learning problem solving, competitions, training talented students.

ZDM Subject Classification: D50, U40.

\section{Introduction}

For 20 years in every year the so called "Nagy Károly Mathematical Studentmeetings" have been organized in Rév-Komárom. Their establisher and organizer is György Oláh, a teacher of mathematics from Rév-Komárom. These Studentmeetings are continuations of the traditions which were initiated in the reformera by Károly Nagy (1797-1868) academic polymath. They mediate the message of Károly Nagy's mathematical activity. In 1835 he won the Golden Price of the Hungarian Academy for his Hungarian mathematical books written for the talented and diligent students. He edited the Hungarian version of Babbage's seven digit logarithmical table in 1834 in London.

Copyright (C) 2012 by University of Debrecen 


$$
\text { "tmcs-kantor" — 2012/2/27 - 20:51 — page } 78 \text { - \#2 }
$$

For these three days' mathematical training sessions the students come mainly from the Upperland (Slovakia), in lesser part from Hungary, rarely from Transylvania (Romania), Sub-Carpathia (Ukraine) and the South (Serbia). Their mother tongue is Hungarian. On the training sessions teachers of mathematics prepare them how to solve mathematical contest problems.

On the 10th anniversary of the "Nagy Károly Mathematical Student-meetings" a booklet [6] was published, containing an appreciation of the events, the programs of the former years and the abstracts of the current training sessions. After it every year a booklet appeared, containing the actual abstracts. These booklets were edited by us (S. and T. Kántor). We took part on 13 series of training sessions, so this article is based on our personal experience too. We outline the values which enrich the theory and practice of the training of talented students. From the abundance of the rich topics we could pick out only a moderate choice. We have to mention that during the 20 years 138 teachers presented 632 lessons.

Most of the lessons of the training sessions were characterized by such kinds of problem-fields which had common topics or solving methods. The material is especially appropriate for preparing students for mathematical competitions. On the occasion of the 20th year a book was published written by 23 teachers [4]. They described one of their lectures from the former years, the posed problemfields and detailed the solutions and proofs.

First we present (Problems I.) a problem each of the 17 teachers who took part and held lectures at more than half of the "Nagy Károly Mathematical Studentmeetings" (i.e. at least on 11 occasions) in alphabetical order and then hints to the solution of these problems. Then we present and comment the topics of 6 teachers whose topic or problem-field were unusual, individual and very valuable for the theory of talent training in spite of their having less activity (Problems II.).

The most important feature of the talent training on the "Nagy Károly Mathematical Student-meetings" is the practicing of the mathematical thinking and knowledge. Each teacher in his or her own way, according to his or her individuality and interests chooses the most adequate one from his or her mathematical topics, but the point is always the same: they want to teach the way of mathematical thinking of the connected theory and practice.

In our article the detailed statements concern the theory and practice of the training of talented students. They are part of the mathematical thinking and understanding or they concern special cases. Most of them are not new but it is worthwhile to write and to analyze them because they are the gems of these
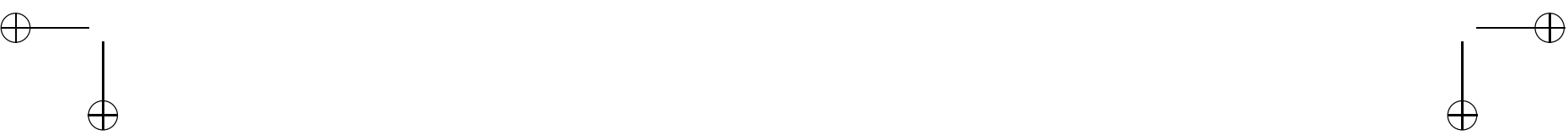


$$
\text { "tmcs-kantor" — 2012/2/27 — 20:51 — page } 79 \text { — \#3 }
$$

training sessions and we can very well apply them for the instruction of younger mathematicians.

\section{Didactical statements (E1 - E8.)}

E1. In the process of talent training we have to make the students think. The role of the conceptual and procedural memory is not significant. Even the text of these problems makes it obvious that they want to make the students think. They are precisely composed and their topics and questions are not from the customary school-mathematics.

E2. Dealing with mathematics requires creativity. Solving the problems is not automatic and it is not only the application of classroom mathematics. The solutions often need a lot of complementary knowledge or methods. We shall emphasize the special cases of creativity in the samples.

E3. The special mathematical thinking is connected with the words always, never, at least, at most, and so on. We remark, that these words were first introduced by Károly Nagy in 1834 in a Hungarian book.

In other sciences we do not find such kinds of everlasting statements as in mathematics, as for example: 1 . The points which we can construct by the help of a ruler and a compass we can always construct with a compass only. 2. There does not exist a radix formula by the help of which all of equations of degree 5 are solvable.

E4. The sensitiveness for mathematical problem posing and the finding of new methods are developed by the searching of not usual objects between the well known concepts.

E5. The mathematically talented student oversteps the limit of his/her age and preparations. Maybe he/she does not understand all details, but understands the essence of the problems in younger age with less preliminary knowledge.

E6. The mathematically talented students are motivated by the problems of mathematical thinking. The abstract concepts, also unthinkable situations in practice, are interesting and exciting for them if they make them think. They enjoy logical challenge.

E7. The mathematically talented students often solve problems which belong to higher mathematics. Their preparation for the contests means that we have to instruct them in some topics and methods from higher mathematics. We 


$$
\text { "tmcs-kantor" — 2012/2/27 - 20:51 — page } 80 \text { - \#4 }
$$

could often observe that the knowledge of previous mathematical contests from higher mathematics became part of school-mathematics.

E8. The excellent leader-professors of mathematical sessions of talented students often recommend to take problems from research work and pose such kind of problems in a simple form (G. Polya, T. Szele). The contest problems given for the students of the universities are of such kind (for example: Schweitzer Contest).

\section{Mathematical statements}

We will discuss statements of mathematical character by the concrete analysis of problems, emphasizing the mathematical content. It is especially important at the more difficult problems because it is not sufficient to know classroom mathematics to solve these. We will denote with letters $\mathrm{K}$ the new knowledge and with letters $\mathrm{M}$ the new methods and we supply them with serial numbers too.

We remark that the hints on E6. connected with classroom mathematics and competition problems are often mixed. The topics are not completely new to the students but the emphasis might have been different. It is easy to see whether some notions are part of the solution and are well known or maybe they are part of the solution and it is necessary to find them out or to prove them.

\section{Problems I.}

Problem 1. Cut a triangle with a straight line in two parts with equal perimeters and areas. Construct this straight line.

Zoltán Bogdán

This problem is a typical case of E4. We often use this method in solving problems.

M1. Principle: "Divide into parts". There are two conditions which have to be satisfied. First we choose one of the conditions and from the objects which satisfy the first condition we will choose those which satisfy the second condition. The proof of the problem might be subdivided into two parts. We can follow Polya's principle: "Find a simple related problem and solve it."

We denote the edges of the given triangle $A B C$ so that $\overline{A B}>\overline{A C}$, and $\overline{A A_{1}}$ is a median. Fit point $E$ to $\overline{A B}$ and point $F$ to $\overline{C A_{1}}$, so that $\overline{E F}$ divides equally 


$$
\text { "tmcs-kantor" — 2012/2/27 - 20:51 — page } 81 \text { - \#5 }
$$

the perimeter of the triangle $A B C$. If $\overline{E A_{1}}$ was parallel to $\overline{A F}$ then $\overline{E F}$ should divide the area equally in consequence of a simple theorem:

K1. A trapezium is cut by its diagonals into four triangles from which the area of those two for which one side is a leg of the trapezium are equal. In consequence, dividing the area equally means that $\overline{A E}-\overline{F A_{1}}=\frac{1}{2}(\overline{A B}-\overline{A C})$ is given, so we can construct $E$ and $F$ by computing $\overline{F A_{1}}$.

Problem 2. Prove that it is not possible to cut a cube with a plane in an obtuse triangle.

Ferenc Csorba

The three-dimensional problems are not difficult, but they are often unfamiliar to many students. It is not obvious which reasoning will be acceptable for the teachers and which arguing or descriptiveness will suit the students.

First we prove that section of a cube in a triangle is possible only when the plane cuts three vertices adjacent to the same edge of the cube. The second part is trivial if we apply the following pretty remark:

K2. A triangle is an acute triangle if and only if the square sum of two arbitrary sides is more than the square of its third side.

Remark: Problem 2 was solved with an unusual new method by the problem poser. He used the scalar product of vectors.

Problem 3. We have to transport 50 boxes from a store. The masses of the boxes are in order $145,146, \ldots, 193,194 \mathrm{~kg}$. We could rent a truck with $1060 \mathrm{~kg}$ carrying capacity. How many turns will be necessary at least? Gábor Erdös

This problem is a brilliant example to show how is it possible to compose a very compact every day problem shortly and completely unambiguously. It is obvious that such kind of distribution of masses of boxes is hardly imaginable in the every day context, but a similar case is believable. We do not feel that Problem 3 is an artificial problem. It is a good example of E2.

M2. For the solution we need systematic trials, a creative work, observing and examination of lot of conditions and outworking the details (strategy: trial and errors). In one round we have to transport at least 6 , and at most 7 boxes. If we organize 8 turns then in 2 cases we have to transport 7 boxes. But this case is not possible in consequence of the mass limit. In 9 turns the transportation is possible. 


$$
\text { "tmcs-kantor" — 2012/2/27 - 20:51 — page } 82 \text { - \#6 }
$$

Problem 4. Let point $D$ be the foot point of altitude $m_{c}$ of the acute triangle $A B C$, and point $V$ the orthocentre of triangle $A B C$. Prove that $\overline{A D} \cdot \overline{B D}=$ $\overline{A B} \cdot \overline{V D}$ is true iff $\overline{C D}=\overline{A B}$.

Pál Hornyák

Remark The word "iff" is part of mathematical logic/thinking.

K3. For the solution it is necessary to know the theorem of secant and segments. If we remember them we can easily prove the statement. But the first step is to use the method of geometrical transformations, reflection on a straight line.

Problem 5. Into how many parts at most is a plane cut by $n\left(n \in \mathbb{N}^{+}\right)$ straight lines?

István Hortobágyi

The topic of Problem 5 is combinatoric geometry. Nowadays some of its parts belong to classroom mathematics. There is a rich generalization of this problem: Into how many parts at most is the space divided by $n\left(n \in \mathbb{N}^{+}\right)$planes?

M3. We solve the problem by the help of mathematical induction (extremal principle). In instructing mathematically talented students it is very useful to teach new mathematical topics, new concepts and new problem solving methods (see E7.).

Problem 6. Let be given tetrahedron $A B C D$. How do we have to choose plane $S$ so that its intersection with tetrahedron $A B C D$ is a parallelogram? In which case would this intersection be a rectangle? In this case, is it possible to get a square as an intersection?

Erzsébet Kalácska

M4. The teaching of space geometrical problems requires special methods. One of the best methods is to develop problem-fields and let the students solve these problems connected with each other. Here the teacher did so. We presented one excellent problem of the problem-field.

Problem 7. A point $P$ is given in the interior of a parallelogram. Construct a straight line through the point $P$ which cuts from the parallelogram the smallest area!

József Kalácska

M5. In the process of getting acquainted with the posed problem it is useful to collect the properties, knowledge, cognitive methods concerning it (Experimentation). 


$$
\text { "tmcs-kantor" — 2012/2/27 — 20:51 — page } 83 \text { - \#7 }
$$

K4. Experimenting on special cases promotes finding of the solutions, therefore it is useful to begin the solving process with analyzing special cases. In classical plane geometry there are some common ideas and characteristic methods. If we deal with triangles, polygons, parallelograms and trapeziums we have to know that geometrical transformations (for example reflections) are very useful tools.

If point $P$ is the centre of symmetry of a parallelogram then its diagonals cut it into two parts with equal areas. We choose the parallel sides of the parallelogram to which point $P$ is nearer. We reflect on point $P$ the sides of the parallelogram. Thus we obtain a parallelogram whose centre is $P$. On the basis of the special case we get that one of the diagonals of the parallelogram is the appropriate straight line.

Problem 8. We suppose that the definitions of the magnitude of vectors, sum and multiplication of vectors by a scalar are known. We assume that the scalar product of two vectors is a real number, and for arbitrary vectors $\vec{a}, \vec{b}$, $\vec{c}$, real number $x$ and $|\vec{e}|=1$ the following properties are satisfied:

$$
\vec{a} \vec{b}=\vec{b} \vec{a}, \quad \vec{a}(\vec{b}+\vec{c})=\vec{a} \vec{b}+\vec{a} \vec{c}, \quad x(\vec{a} \vec{b})=(x \vec{a}) \vec{b}, \quad \vec{e} \vec{e}=1 .
$$

Prove that $\vec{a} \vec{b}=|\vec{a} \| \vec{b}| \cos (\vec{a}, \vec{b})$.

Sándor Kántor

Problem 8 is a so-called characterization theorem for the scalar product of vectors. Professor T. Szele posed this theorem as a problem for younger mathematicians. As a student (age 15) S. Kántor heard it from him [1]. Later he recognized that T. Szele proved this theorem for vectors of higher dimensions. It was acknowledged as a scientific result (see E7., E8.).

K5. The content of the definition is consequence of some properties of the notion to be defined.

M6. Theorems of characterization are a special part of mathematics. We make the proof first for special cases thus simplifying the problem, dividing it into parts and at the end we turn to the generalization (Principle: specializing and generalization). The special cases are: $\vec{a}$ and $\vec{b}$ are parallel and of the same direction; $\vec{a}$ and $\vec{b}$ are parallel and of the opposite direction; $\vec{a}$ and $\vec{b}$ are perpendicular to each other.

Problem 9. For how many positive integers $n$ does $1+2+\cdots+n$ evenly divide $6 n$ ? Choose the answer from the following marked letters (only one answer
is correct):
(A) 3
(B) 5
(C) 7
(D) 9
(E) 11
Tünde Kántor 


$$
\text { "tmcs-kantor" — 2012/2/27 - 20:51 — page } 84 \text { - \#8 }
$$

This problem was posed on the 6th AMC 10 A (American Mathematics Competition, 2005). In our case it was motivated by the test character and from the source. In solving test-problems it is very important that time is limited. The students have to work quickly and correctly. The correct answer is B, because $n+1$ has to divide 12 .

M7. Tests contain many, sometimes simple, problems. The main task is to select the wrong answers quickly and to find the possible good solution in limited time. There are a lot of methods for solving tests [2]. These abilities are useful also in traditional competitions in contest-problem solving.

Problem 10. Let us denote by $E$ the midpoint of the side $B C$ of square $A B C D$ and by $F$ the foot-point of the perpendicular drawn from point $A$ to $D E$. Prove that the triangle $A B F$ is isosceles.

Károly Károlyi

M8. The method of solution of a geometrical problem is the following: sketch figures and complete them with auxiliary lines [7]. It is very important to recognize some familiar features in the given geometrical figure (Principle: Guess and prove). In the case of Problem 10 it is suitable to sketch the height $B B^{\prime}$ of the triangle $A B F$ from the point $B$. Then it is easy to discover that $B B^{\prime}$ is a bisector too.

Problem 11. A positive integer with at least two digits is called an increasing number if its digits (from left to right) form a strictly increasing sequence. How many increasing numbers do there exist the sum of whose digits is 30 ?
(A) 13
(B) 14
(C) 15
(D) 16
(E) 17
István Keszegh

Problem 11 was posed on the "Zrínyi Ilona Mathematical Competition" (2005) for pupils grade 8. (age 14).

M9. To solve a counting problem quickly it is sometimes better to apply the method of counting down complementary cases, i.e., cases which do not satisfy the conditions. In solving test-problems it is important to minimize the time. Now we get the complementary cases by deleting digits from the increasing number 123456789 , containing all digits in such a way that the sum of the rest is 30 , i.e. by deleting digits whose sum is 15 . The number of such digit-groups is 17 . The right answer is: (E).

Problem 12. Let be $A_{1}, A_{2}, \ldots, A_{n}$ a regular polygon with $n$ odd edge numbers, and $M$ a point of $A_{1} A_{n}$, the arc of its circumcircle. Prove that the sum 


$$
\text { "tmcs-kantor" — 2012/2/27 — 20:51 — page } 85 \text { — \#9 }
$$

of the length of lines connecting $M$ to vertices with odd serial numbers is equal to the sum of the length of lines connecting $M$ to vertices with even serial numbers.

Antal Kubatov

If we see only Problem 12 from the presented problem-field then it is hard to imagine that it is connected with the Theorem of Ptolemy. In geometry it is useful to know and apply a lot of general theorems. The contest problems are often special cases of some better or less known theorems. Solution of this problem is a brilliant example how to connect methods and knowledge and to deepen students horizon.

Problem 13. Six irrational numbers are given. Is it true that among them there exist three from which the sum of any two numbers irrational?

László Laczkó

M10. The introduction of illustration with graphs makes easier the visualisation and description of the solution (Principle of perceptive variety). Let the given irrational numbers be the vertices of a graph. Two vertices are connected with an edge if the sum of the two irrational numbers is irrational. It is easy to see that in this graph there is no complete graph with three edges. From these facts the statement follows.

Problem 14. Find the minimum of the product $(x+y)(x+z)$, if $x, y, z>0$, and $x y z(x+y+z)=1$.

The words and the formulas are usual, but there are too many products. We are afraid that the solution will be very complicated. We are exited about finding simplifying idea.

M11. It is necessary to replace the problem with a more perspicuous one, when the formulas are complicated or the text is too long (Looking for a specialization). Problem 14 reduces in consequence of the conditions to examining the sum of a number and its reciprocal. In this way we get a very nice and elegant solution.

K6. It is easy to find the minimum on the basis of the formula:

$$
(x+y)^{2}-(x-y)^{2}=4 x y .
$$




$$
\text { "tmcs-kantor" — 2012/2/27 — 20:51 — page } 86 \text { — \#10 }
$$

PROBLEM 15. In a number written in the decimal system there are 300 one digits and the other digits are equal to 0 . Is it possible that this number is a perfect square?

György Oláh

Problem 15 was posed on the "Arany Dániel Mathematical Competition" (1975) on advanced level for pupils grade 10. (age 16).

The concrete number 300 is a special case. For the solving of Problem 15 it is necessary to recognize that

K7. If a perfect square number is divisible with 3 it is divisible with 9 too.

PROBLEM 16. Let $k$ and $d$ be positive integers. Prove that, if there is a perfect square in the sequence $k, k+d, k+2 d, k+3 d, \ldots$, then there are infinitely many perfect squares.

Ferenc Pintér

In solving Problem 16 we use method of guess and prove. The concept of infinity is not familiar to the students. To solve the problem students have to be creative. They have to guess: if $k+n d=x^{2}$, then $(x+d)^{2}=k+(n+2 x+d) d$ will be a new and bigger perfect square in the sequence.

M12. The method is an application of a new mathematical concept, the concept of the potential infinity (without naming it).

Problem 17. The difference of the cubes of two consecutive positive integers is $n^{2}\left(n \in \mathbb{N}^{+}\right)$. Prove that $n$ is the sum of two perfect squares. János Urbán

Problem 17 has a story. First in 2010 it was posed on the regional round of the "Kalmár Competition" ( grade 8, age 14). So we could think that it is not a hard task. Later it was posed again in the Hungarian Mathematical and Physical Journal for Secondary Schools (KöMal, 2010. No. 7, B 4301). In KöMal it got a relatively high score, that means it is not too easy. The third time it was posed in 2010 on the "Nagy Károly Mathematical Student-meeting".

K8. The knowledge of the form of Pythagorean triples is expected from contestants. Realizing that if $n^{2}=(a+1)^{3}-a^{3}$ then $(2 n+1)(2 n-1)=3(2 a+1)^{2}$ we get immediately the statement (Guess and prove). 


$$
\text { "tmcs-kantor" — 2012/2/27 — 20:51 — page } 87 \text { — \#11 }
$$

\section{Problems II.}

Problem 18. For which values of the parameter $p$ have the following equations (separately) exactly one solution?

$$
\begin{aligned}
& \sqrt{x-1}=x-p \\
& x+1=\sqrt{p x} .
\end{aligned}
$$

András Ambrus

The simplest way of the solution is to draw the graphs. The place of the visual information is in the right half of our brain.

M13. The interpretation is connected with the structure of the human brain. We have to use our cerebral hemispheres!

Problem 19. There are given in the plane $n>2$ points. Does there exist such a straight line to which at least two of them fit and in the half planes determined by this straight line the number of the points of the given point set differs maximum by two?

Béla Bálint

M14. Problem 19 seems to be simple, but till today it is unsolved. These kind of problems are exciting and challenging for contestants.

Problem 20. The sequence $a_{1}, a_{2}, a_{3}, \ldots$ is defined by the formula $a_{n}=$ $2^{n}+3^{n}+6^{n}-1\left(n \in \mathbb{N}^{+}\right)$. Determine all positive integers which are relative primes to all elements of the sequence.

Sándor Dobos

Problem 20 was posed on IMO in 2005. S. Dobos, as one of the companion of the Hungarian IMO team, presented every year actual IMO problems on the "Nagy Károly Mathematical Student-meetings", spiced with his personal experiences.

M15. Presentation of higher, but not too hard problems is important in the education of students with high ability.

K9. A knowledge of the little Fermat Theorem is expected.

Problem 21. Connections between mathematics and music (Fibonacci numbers).

Csabáné Iharos
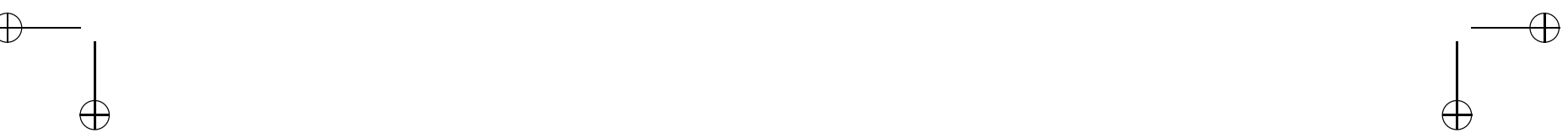


$$
\text { "tmcs-kantor" — 2012/2/27 — 20:51 — page } 88 \text { — \#12 }
$$

M16. Presentation of connections between different fields is very interesting and attractive for the students.

Problem 22. Prove that if two straight lines are perpendicular to a third one, then these two lines are not intersecting. For the arguing do not use the axiom of parallels.

Attila Kálmán

M17. The topic of Problem 22 is absolute geometry. It is a new and exciting topic for students (E7.).

Problem 23. The lengths of segments $a, b$ and $c$ are given.

Construct by the help of compass only the segment with the length $x$ if $a: b=c: x !$

Géza Kiss

Problem 23 is a special case of Mascheroni's famous theorem: any geometrical construction which is possible with compass and straight ruler also can done using compass only. Here we have to construct the proportion of the fourth.

We quickly get the solution using concentric circles of radius length $a$ and $b$ (or $a: b)$.

M18. Posing Problem 23 is an important case of dealing with research problems (E8.).

\section{References}

[1] S. Kántor, Die Methode von Prof. Tibor Szele im Unterricht begabter Schüler, Teaching Mathematics and Computer Science 4, no. 1 (2006), 143-151.

[2] T. Kántor, Mathematics tests in PISA 2003, Practice and Theory in Systems of Education 3, no. 1 (2008), 55-62, ISSN 1788-2591.

[3] T. Kántor, Anatomy of a contest problem Pro. Math. Debrecen 2005, Problem Solving in Mathematics Education, Proceedings of the ProMath 6 Meeting, (T. Kántor, ed.), Institute of Mathematics University of Debrecen, Hungary, 2006.

[4] G. Kiss (ed.), Calculation, (Hungarian: Szám-vetés), Zalamat Alapítvány Nagykanizsa, 2010.

[5] M. Laczkovich, Guess and Prove, (Hungarian: Sejtés és Bizonyítás), TypoTex, 1998.

[6] G. Oláh (ed.), The first 10 years of the Nagy Károly Mathematical Student-meetings (1991-2000) in documents, (Hungarian: A Nagy Károly Matematikai Diáktalálkozó egy évtizede (1991-2000) dokumentumokban), University of Debrecen, 2000.

[7] G. Polya, Mathematical Discovery on Understanding, Learning, , Teaching Problem Solving I-II., New York, John Wiley Sons, 1962, 1965. 
"tmcs-kantor" — 2012/2/27 — 20:51 — page 89 — \#13

[8] G. Polya, Mathematics and Plausible Reasoning I-II., Princeton University Press, 1954.

[9] I. Reiman and S. Dobos, International Mathematics Olympiads (Hungarian) 1959-2003, TypoTex, 2003.

SÁNDOR KÁNTOR

DEPARTMENT OF ANALYSIS

UNIVERSITY OF DEBRECEN

HUNGARY

E-mail: kantor.sandor@science.unideb.hu

TÜNDE KÁNTOR

DEPARTMENT OF GEOMETRY

UNIVERSITY OF DEBRECEN

DEBRECEN

E-mail: tkantor@science.unideb.hu

(Received April, 2011) 